Report

\title{
Pharmacological characterization of 4-hydroxy- $N$-desmethyl tamoxifen, a novel active metabolite of tamoxifen
}

\author{
Michael D. Johnson ${ }^{1}$, Hong Zuo ${ }^{1}$, Kyung-Hoon Lee ${ }^{2}$, Joseph P. Trebley ${ }^{3}$, James Michael Rae ${ }^{4}$, \\ Ross V. Weatherman ${ }^{2}$, Zeruesanay Desta ${ }^{2}$, David A. Flockhart ${ }^{2}$, and Todd C. Skaar ${ }^{2}$ \\ ${ }^{1}$ Department of Oncology, Lombardi Comprehensive Cancer Center, Georgetown University Medical Center, \\ Washington, DC; ${ }^{2}$ Division of Clinical Pharmacology, Department of Medicine, Indiana University School of \\ Medicine, Indianapolis; ${ }^{3}$ Department of Medicinal Chemistry and Molecular Pharmacology, Purdue University, \\ West Lafayette, IN; ${ }^{4}$ Division of Hematology and Oncology, Department of Internal Medicine, University of \\ Michigan Medical Center, Ann Arbor, MI, USA
}

Key words: endoxifen, estrogen receptor, gene expression, metabolite, proliferation, receptor binding, tamoxifen

\section{Summary}

The antiestrogen tamoxifen is extensively metabolized in patients to form a series of compounds with altered affinity for estrogen receptors (ERs), the primary target of this drug. Furthermore, these metabolites exhibit a range of partial agonist and antagonist activities for ER mediated effects that do not depend directly on their absolute affinity for ERs. Thus, clinical response to tamoxifen therapy is likely to depend on the aggregate effect of these different metabolites resulting from their abundance in the patient, their affinity for the receptors, and their agonist/antagonist profile. A recent study has shown that plasma concentrations of the tamoxifen metabolite 4-hydroxy- $N$-desmethyl tamoxifen (endoxifen), in patents undergoing tamoxifen therapy, are dependent on the cytochrome P450 (CYP) 206 genotype of the patient and that medications commonly prescribed to patients on tamoxifen therapy can also inhibit endoxifen production. In this study we characterized the properties of this metabolite with respect to binding to ERs, ability to inhibit estrogen stimulated breast cancer cell proliferation and the regulation of estrogen responsive genes. We demonstrate that endoxifen has essentially equivalent activity to the potent metabolite 4-hydroxy tamoxifen (4-OH-tam) often described as the active metabolite of this drug. Since plasma levels of endoxifen in patients with functional CYP2D6 frequently exceed the levels of 4-OH-tam, it seems likely that endoxifen is at least as important as 4-OH-tam to the overall activity of this drug and suggests that CYP2D6 status and concomitant administration of drugs that inhibit CYP2D6 activity have the potential to affect response to tamoxifen therapy.

\section{Introduction}

It is estimated that in 2002 there were 203,500 women newly diagnosed with breast cancer and that 39,600 woman died of the disease [1]. Roughly 60-65\% of these women will have tumors that are estrogen receptor (ER) positive. Of ER positive tumors, approximately $60-65 \%$ will respond to some form of hormonal therapy. Since its introduction 30 years ago, tamoxifen has been the front line hormonal therapy of choice [2]. With the additional use of tamoxifen in the adjuvant setting, and now with evidence that it is an effective chemo-preventive agent, it is not surprising that tamoxifen is the most commonly prescribed drug in the management of breast cancer. Tamoxifen is a selective estrogen receptor modulator (SERM) - that is a compound which competes with estrogen for binding to the ER and exhibits a spectrum of estrogen antagonist and agonist activity depending on the site of action. In the breast it is primarily an antiestrogen and it is believed to inhibit tumor growth by starving the tumor cells of survival and proliferative signals provided by estrogen signaling.

Tamoxifen is sometimes described as a pro-drug, since it is extensively metabolized yielding several compounds that are more potent antiestrogens than 
the parent compound [3]. However, tamoxifen is quite active itself and due to the relatively low concentration of the more potent metabolites, it seems likely that the overall response of the tumor is the result of the aggregate effect of the drug and its metabolites. Interestingly, some minor tamoxifen metabolites are potent estrogens and many investigators have suggested that different patterns of metabolism of the compound among patients might go some way to explaining why only approximately $60 \%$ of ER positive tumors respond to tamoxifen therapy.

We recently reported the identification of a tamoxifen metabolite (4-hydroxy- $N$-desmethyl tamoxifen endoxifen), the plasma concentration of which in patients receiving tamoxifen is altered by concomitant administration of paroxetine [4]. Furthermore, we showed that plasma concentrations were dependent on the genotype of the patient with respect to polymorphisms of an enzyme known to metabolize both paroxetine and tamoxifen (cytochrome P450 2D6 CYP2D6) [5]. In this study we present a detailed characterization of the ER binding properties of this metabolite and its effects on the expression of estrogen responsive genes and on the proliferation of ER positive breast cancer cells.

\section{Methods}

All chemicals were from Sigma (St. Louis, MO) unless otherwise noted.

\section{Synthesis and purification of endoxifen}

Endoxifen for use in this study was synthesized using two separate methods. The first method is described in our previous paper [4]. The second method was developed to facilitate the synthesis of large amounts of the compound for in vitro and in vivo characterization and is summarized in Figure 1. The product of each reaction was purified using silica gel chromatography. Endoxifen was purified following the final reaction using silica gel chromatography with 90:9: $1 \mathrm{CHCl}_{3} / \mathrm{CH}_{3} \mathrm{OH} / \mathrm{NH}_{4} \mathrm{OH}$ as the eluent. Using ${ }^{1} \mathrm{H}$ NMR, ${ }^{13} \mathrm{C}$ NMR and high resolution mass spectrometry, the identity of the final product was confirmed as a $1: 1$ mixture of $E$ and $Z$ regioisomers of endoxifen (spectra are available upon request). Endoxifen prepared by either method produced identical results in the assays described below.

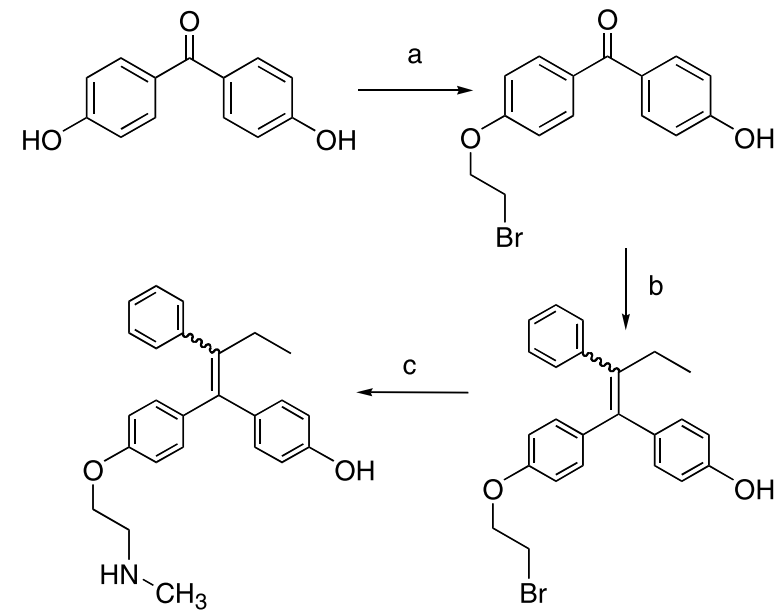

Figure 1. Synthetic scheme for the production of endoxifen. (a) $\mathrm{KH}$, bromoethane, THF, DMF, reflux, $17 \%$ yield; (b) propiophenone, $\mathrm{TiCl}_{4}, \mathrm{Zn}$ dust, THF, reflux, $65 \%$ yield; (c) $\mathrm{CH}_{3} \mathrm{NH}_{2}$ in $\mathrm{MeOH}$, $85^{\circ}, 70-90 \%$ yield.

\section{Cell culture}

MCF-7, T47D and BT474 cells were obtained from the tissue culture shared resource (TCSR) at the Lombardi Comprehensive Cancer Center and were routinely cultured in modified IMEM supplemented with $10 \%$ fetal calf serum (Life Sciences, Gaithersburg, MD).

Proliferation assays. Cell monolayers at about $70 \%$ confluence were withdrawn from estrogen by changing to an estrogen-depleted medium consisting of phenol red-free modified IMEM supplemented with charcoal stripped newborn calf serum. To remove residual estrogen the monolayer was washed every hour for $5 \mathrm{~h}$ with phenol red-free IMEM and cultured in fresh medium in between washes. The cells were then cultured overnight and trypsinized with phenol redfree trypsin. The cells were counted and 1000 cells plated into each well of several 96 well plates in $100 \mu \mathrm{l}$ of the same medium. After allowing the cells to attach $(5 \mathrm{~h}), 100 \mu \mathrm{l}$ of medium containing $17-\beta$-estradiol (E2), endoxifen, or 4-OH-tam (alone or in combination with E2), or the vehicle control (ethanol), were added to the wells to yield the indicated concentrations of the various compounds and a final ethanol concentration of $0.1 \%$. Several duplicate plates were prepared and after allowing the cells to grow for various periods, the cells were stained with crystal violet $(0.52 \%$ crystal violet in $25 \%$ methanol). After washing the plates to remove excess stain, the plates were dried and 
then the bound stain solubilized by addition of $100 \mu 1$ of $100 \mathrm{mM}$ sodium citrate in $50 \%$ ethanol. Staining intensity, which is proportional to cell number, was then determined by measuring absorbance at $570 \mathrm{~nm}$ using a 96 well plate reader [6].

\section{ER binding assays}

The binding of endoxifen to the ERs was investigated by competition binding assays using two different methods. The dextran coated charcoal method and a fluorescence polarization method were used to evaluate binding to ER- $\alpha$, and the fluorescence polarization method was used to investigate binding to ER- $\beta$.

Dextran coated charcoal assay. Competitive binding assays were conducted using a modification of a standard method [7]. Briefly, MCF-7 cells were withdrawn from estrogen by washing and growth in depleted medium (as described above) for 3 days to maximize ER levels. The cells were harvested with phenol redfree trypsin (Biofluids, Rockville, MD) washed with TEG buffer (10 mM Tris, pH 7.4, 1 mM EDTA, $10 \%$ glycerol) and re-suspended at approximately 30 million cells per $\mathrm{ml}$ in TEG buffer supplemented with $1 \mathrm{mM}$ dithiothreitol, $0.5 \mathrm{M} \mathrm{NaCl}$ and protease inhibitors $(1 \mathrm{mg} / \mathrm{ml}$ leupeptin, $77 \mu \mathrm{g} / \mathrm{ml}$ aprotinin, $1 \mu \mathrm{g} / \mathrm{ml}$ pepstatin A and $250 \mu \mathrm{g} / \mathrm{ml}$ pefabloc). A whole cell lysate was prepared by homogenization with a teflon/glass homogenizer (40 strokes on ice) followed by centrifugation at $105,000 \times \mathrm{g}$ for $30 \mathrm{~min}$ at $4^{\circ} \mathrm{C}$. The supernatant was removed and the protein concentration measured using the method of Bradford [8]. The protein concentration in the lysate was adjusted to $2 \mathrm{mg} / \mathrm{ml}$ by dilution with the lysis buffer.

Binding reactions were set up on ice in disposable glass culture tubes (Fisher, Pittsburgh, PA). Whole cell lysate $(100 \mu \mathrm{l})$ was mixed with $50 \mu \mathrm{l}$ of TEG buffer containing drugs to yield a final concentration of $1 \mathrm{nM}{ }^{3} \mathrm{H}$ E2 (TRK 322, Amersham, Piscataway, NJ) in the absence or presence of various concentrations of unlabeled E2, endoxifen or 4-hydroxy tamoxifen (4-OH-tam). After an overnight incubation at $4{ }^{\circ} \mathrm{C}$ the unbound steroids were removed by the addition of $500 \mu 1$ of a $0.5 \%$ suspension of activated charcoal in a $0.05 \%$ solution of dextran $(70,000$ average molecular weight), $10 \mathrm{mM}$ Tris, $\mathrm{pH} 8$. The charcoal was pelleted by centrifugation and $500 \mu \mathrm{l}$ of the supernatants from each tube was placed in scintillation vials with $5 \mathrm{ml}$ of Ecoscint A (National Diagnostics, Atlanta GA) and relative levels of binding determined by scintillation counting.

Fluorescence polarization assay. Fluorescent polarization based competition binding assays were conducted to determine the relative affinity of 4-OH-tam and endoxifen for ER- $\alpha$ and ER- $\beta$ using commercially available kits (P2698 and P2700 respectively, PanVera Corp., Madison, WI). Assays were conducted using a modification of the manufacturer's instructions. Briefly, serial dilutions of E2, endoxifen or 4-OH-tam were prepared in ES2 screening buffer $(100 \mathrm{mM}$ potassium phosphate, $\mathrm{pH} 7.4,100 \mu \mathrm{g} / \mathrm{ml}$ bovine gamma globulin) and $10 \mu \mathrm{l}$ of each concentration was aliquoted into four wells of a black 384 well assay plate (3676 Corning, Corning, NY). Ten microliters of a solution containing $20 \mathrm{nM}$ recombinant ER ( $\alpha$ or $\beta$ ), and $2 \mathrm{nM}$ of a proprietary fluorescent ER ligand (Fluormone $^{\mathrm{TM}}$ ) was added to each well. The plate was shaken on a plate mixer and incubated for $5 \mathrm{~h}$ in the dark at room temperature. Fluorescence polarization signals were then measured using a Tecan Ultra fluorometer (Tecan, Durham, NC).

\section{RNA preparation and analysis}

MCF-7 cells were depleted of estrogen for $24 \mathrm{~h}$ as described above and then plated into $25 \mathrm{~cm}^{2}$ flasks (Costar, Corning, NY). The medium was replaced every $24 \mathrm{~h}$ (phenol red free IMEM with $10 \%$ CCS) for 5 days after which the cells were treated with the indicated concentration of endoxifen or 4-OH-tam in the presence or absence of estradiol for 2 or 3 days. The monolayers were washed twice with phosphate buffered saline (PBS) and then solublized in RNAzol (Iso-Tex Diagnostics, Friendswood, TX), and total cellular RNA prepared according to the manufacturers instructions.

Northern blot analysis. Northern blot hybridization was conducted essentially as described previously [9]. Briefly $5 \mu \mathrm{g}$ samples of RNA were fractionated on $1.2 \%$ agarose gels containing $2.2 \mathrm{M}$ formaldehyde and transferred to nylon membranes (Hybond-N, Amersham, Arlington Hights, IL). The uniformity of RNA transfer was verified by examining the ethidiumstained ribosomal RNA bands on the filters under UV illumination. The filters were then hybridized using conditions previously described with riboprobes transcribed from plasmids containing cDNAs for the various genes being studied [9]. After washing, the 

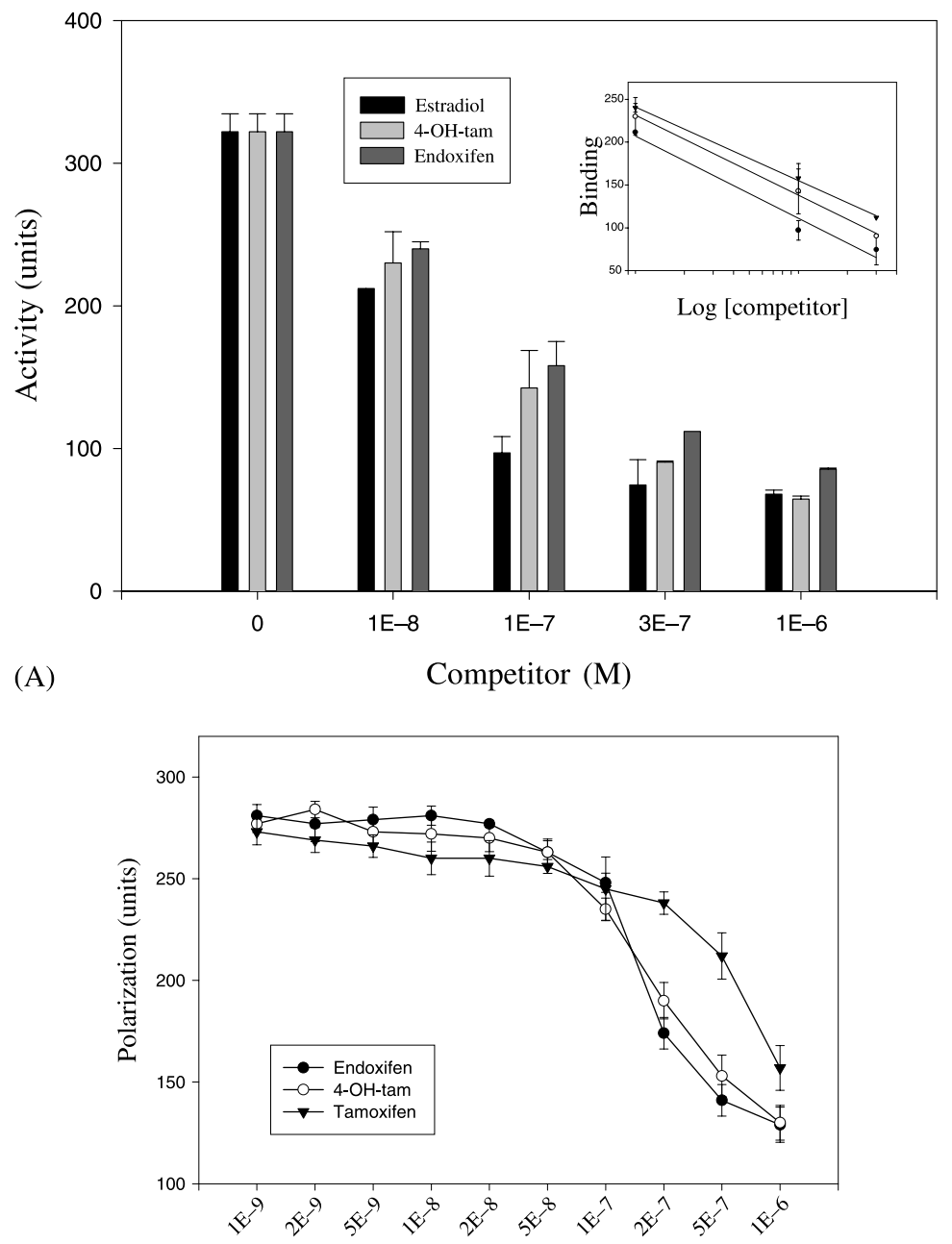

(B)

Antiestrogen (M) plus 2 nM Fluormone ${ }^{\mathrm{TM}}$

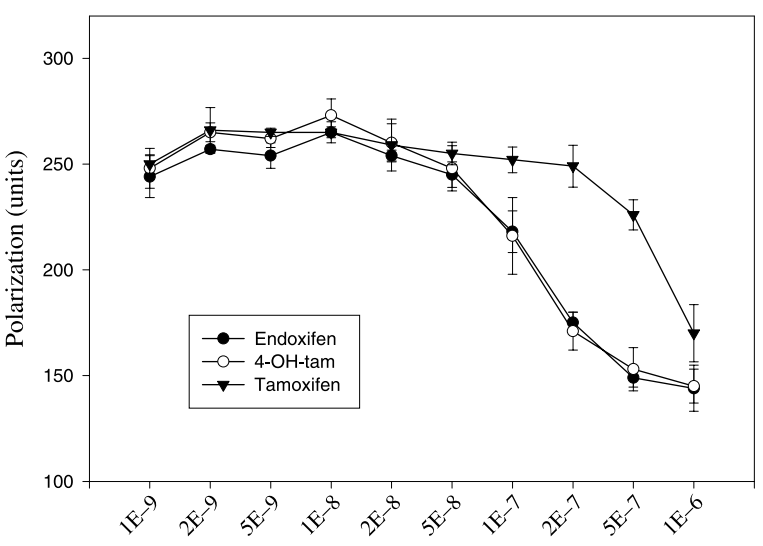

(C)

Antiestrogen (M) plus $2 \mathrm{nM}$ Fluormone $\mathrm{PM}^{\mathrm{TM}}$

Figure 2. Relative ER binding affinity of endoxifen. The ability of various concentrations of endoxifen, 4-OH-tam and estradiol to displace ${ }^{3} \mathrm{H}$ estradiol (A) or a synthetic fluorescent estrogen (B and C) from MCF-7 nuclear extracts (A) or recombinant preparations of the $\alpha$ (B) or $\beta$ (C) form of the ER were evaluated as described in Methods. The mean and standard deviation of at least 2 (A) or 4 (B and C) independent samples are presented for each point. 
radioactive bands on the filters were imaged and quantitated with a phosphorimager (Molecular Dynamics model 445 SI, Sunnyvale, CA). Probes for pS2 and 36B4 were provided by Pierre Chambon (INSERM, Strasbourg, France) and a probe for Cathepsin D was provided by Dr Bruce Westley (University of Newcastle upon Tyne, UK).

\section{Results}

\section{ER binding assays}

To evaluate the ability of endoxifen to bind to the ER, classic radioligand receptor competition binding assays were conducted using cell extracts from ER positive breast cancer cells, as described in the methods section [7]. Figure 2(A) shows data from a representative experiment using MCF-7 cells. Each data point is the mean and standard deviation of two or three samples and each experiment was conducted at least twice. From these data the apparent relative affinity of 4-OH-tam and endoxifen was calculated to be approximately 35 and $25 \%$ that of estradiol, respectively under the experimental conditions used.

To further probe the ER binding characteristics of endoxifen, fluorescence polarization based receptor binding assays were conducted using recombinant human estrogen receptor alpha (ER- $\alpha)$. Comparison of the ability of 4-OH-tam and endoxifen to displace a proprietary fluorescent estrogen from the recombinant ER- $\alpha$ demonstrated that the compounds have an essentially identical affinity for the receptor under these conditions (Figure 2(B)). Similar experiments were conducted to evaluate binding to the beta form of the receptor (ER- $\beta)$, which showed that both compounds had apparently identical affinity for this receptor also (Figure 2(C)).

\section{Proliferation assays}

Assays were conducted to characterize the ER agonist and antagonist properties of endoxifen with respect to the proliferation of several ER positive breast cancer cell lines (MCF-7, T47D and BT474). The proliferation of these cell lines is dependent on estrogenic stimuli and initial studies were conducted to determine the minimum concentration of estradiol required to maximally stimulate the growth of the cells (Figure 3(A)). The dose-response relationship exhibited by all three lines was very similar, with maximal proliferation being produced by stimulation with approximately $200 \mathrm{pM}$ estradiol which was chosen as the dose for subsequent experiments (dotted line in

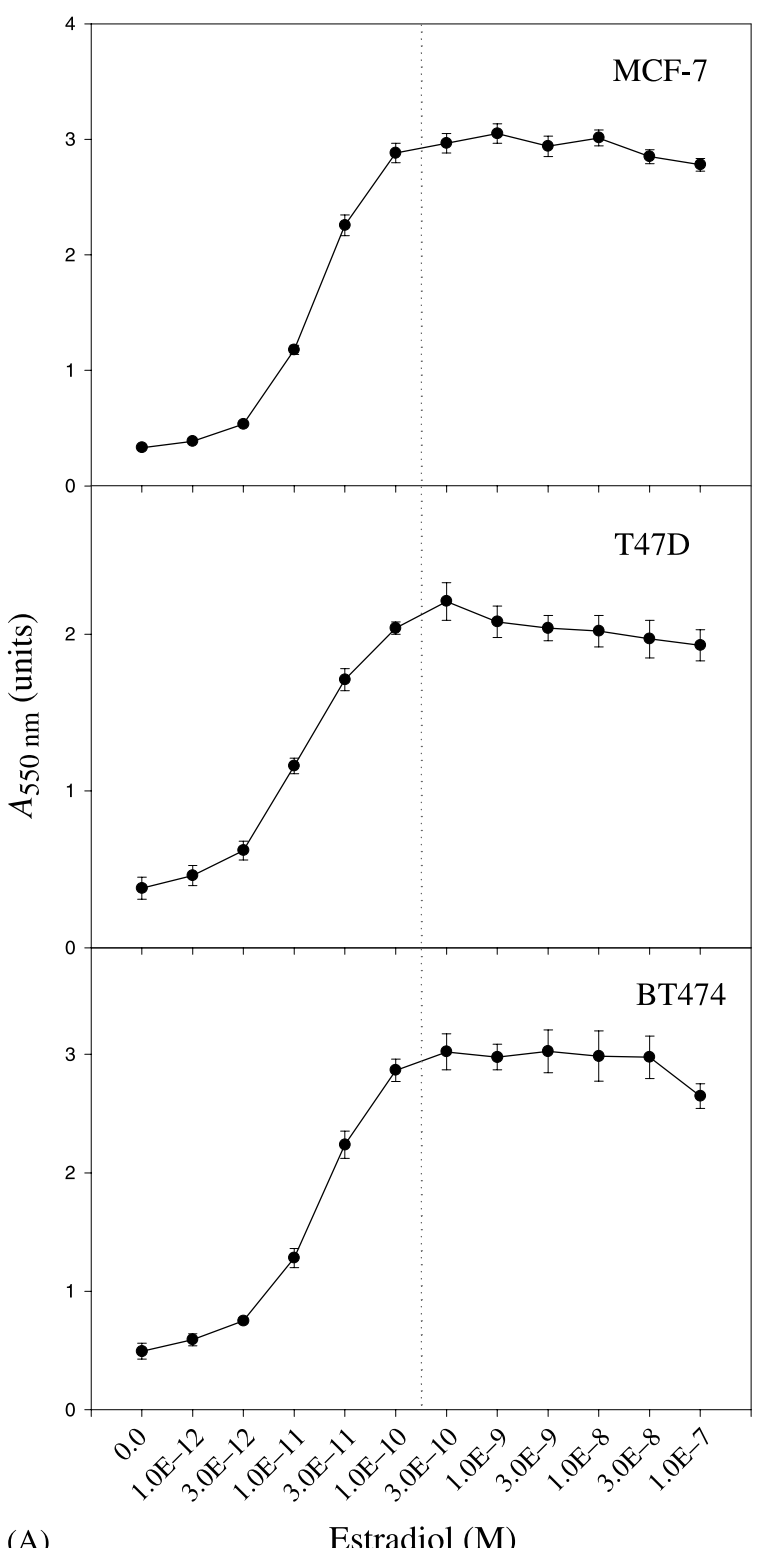

(A)

Figure 3. Inhibition of estradiol stimulated breast cancer cell proliferation by endoxifen and 4-OH-tam. MCF-7, T47D and BT474 cells were withdrawn from estrogen stimulation and treated with the indicated concentration of estradiol for 7-9 days (A). The ability of endoxifen or 4-OH-tam to inhibit the proliferation stimulated by $200 \mathrm{pM}$ estradiol (B) or stimulate cell proliferation in the absence of estrogen $(C)$ was then tested by growing the cells for 7-9 days in the indicated concentration of endoxifen or 4-OH-tam $200 \mathrm{pM}$ of estradiol in the presence (B) or absence (C) of $200 \mathrm{pM}$ estradiol. 


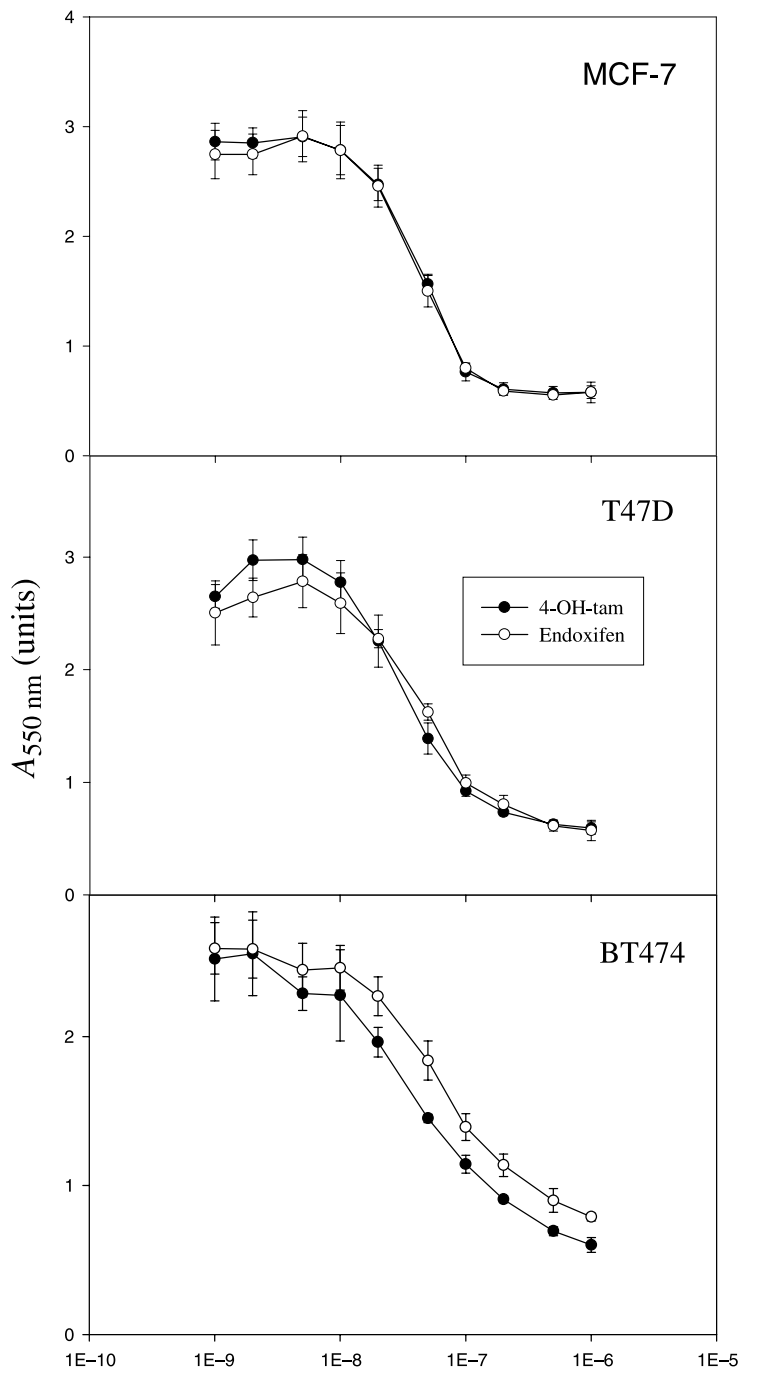

(B) Antiestrogen (M) plus $200 \mathrm{pM}$ Estradiol

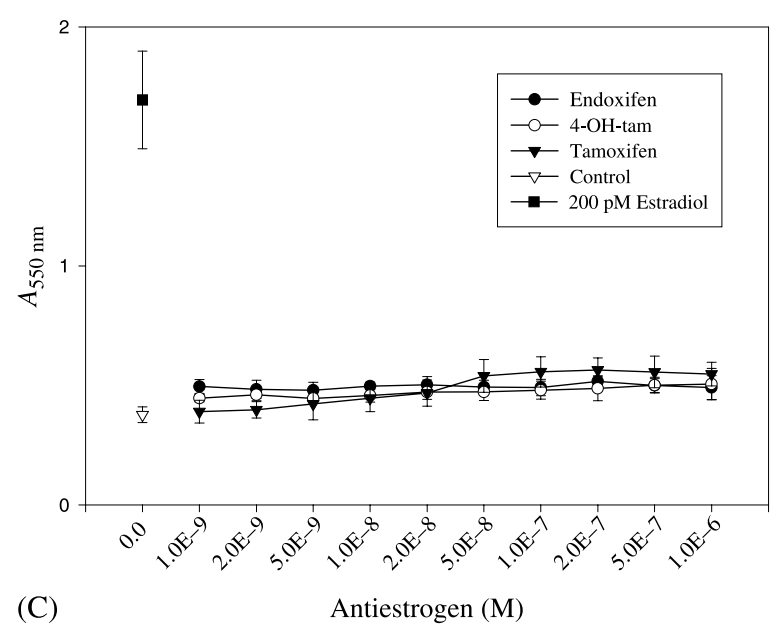

Figure 3. Continued.

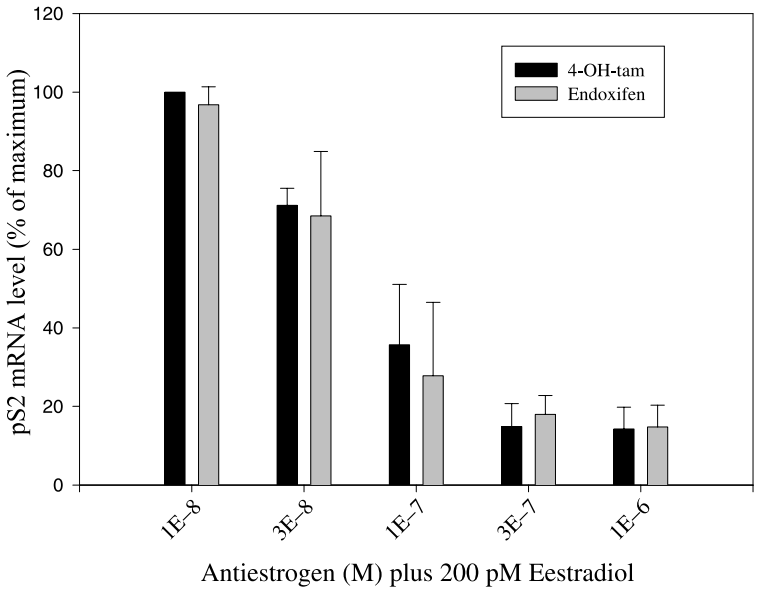

Figure 4. Inhibition of estrogen induced $\mathrm{pS} 2$ expression by endoxifen or 4-OH-tam. Cells were withdrawn from estradiol and treated with $200 \mathrm{pM}$ estradiol in the presence of the indicated concentration of endoxifen or 4-OH-tam for 3 days. pS2 mRNA levels were determined as described in Methods and are expressed as a percentage of maximal pS2 levels normalized to total RNA. The experiment was repeated three times - representative data are presented.

Figure 3(A)). The ability of 4-OH-tam and endoxifen to inhibit the estrogen stimulated growth of the breast cancer cells was examined and both compounds were found to be essentially equipotent, with half maximal inhibition being seen at approximately $50 \mathrm{nM}$ (Figure 3(B)). Both 4-OH-tam and endoxifen were essentially devoid of estrogen agonist activity in this system (Figure 3(C)).

\section{Gene expression experiments}

The effects of endoxifen on the expression of estrogen responsive genes was compared to that of 4-OH-tam by northern blot analysis. The inhibition of $\mathrm{pS} 2 \mathrm{ex}-$ pression by both compounds is shown in Figure 4 . Half maximal inhibition of expression is produced by both compounds between $3 \times 10^{-8}$ and $10^{-7} \mathrm{M}$, consistent with the proliferation data.

\section{Discussion}

In advanced breast cancer, roughly $35 \%$ of patients with ER positive tumors will not respond to tamoxifen therapy, and ultimately all tumors that do respond will become resistant to the treatment, typically after a year to 18 months. Thus, much work has been conducted trying to understand the lack of response 
in tumors that exhibit primary resistance to tamoxifen and the mechanisms by which tumors acquire resistance. One focus of this work has been the possibility that an altered pattern of metabolism might in part explain these effects. Tamoxifen is extensively metabolized, predominantly by the liver, generating a complex series of compounds that exhibit altered affinity for ERs and a variety of agonist and antagonist properties [3,9]. These compounds range in their activity from strong estrogen antagonists to potent estrogens. The most abundant metabolite is $N$-desmethyl tamoxifen (NDT) which is believed to be predominantly generated by the cytochrome $\mathrm{p} 450$ enzyme (CYP) 3A4. Patients receiving the standard does of $20 \mathrm{mg}$ of tamoxifen per day typically have plasma concentrations of NDT of approximately 400$800 \mathrm{nM}$, (compared to $250-650 \mathrm{nM}$ tamoxifen) when they reach steady state, which takes 4-6 weeks since the half-life of NDT is roughly two weeks $[10,11]$. NDT has similar potency to that of tamoxifen and similar partial agonist activity [9]. Another important metabolite is 4-OH-tam, which is sometimes referred to as the active metabolite of tamoxifen, since it is significantly more potent than the parent compound. Depending on the assay system used, the apparent affinity of 4-OH-tam for ER- $\alpha$ is similar to that of E2, whereas that of tamoxifen is approximately 30100-fold less [3]. Plasma concentrations of 4-OH-tam are however quite low, approximately $1-12 \mathrm{nM}$, thus describing 4-OH-tam as the active metabolite is over simplistic, since it is likely that for most patients it is the aggregate activity of the parent compound and metabolites that will determine the response that is seen. Several of the CYPs are known to be able to make 4-OH-tam (CYP2D6, CYP2C9, CYP2C19, CYP3A4) $[4,12]$.

Tamoxifen is generally a very well tolerated drug, however one of the most commonly reported side effects, hot flashes, can be sufficiently severe to lead to patients stopping their therapy [13]. Since treatment with estrogen is not a logical option for treating hot flashes in the context of antiestrogen therapy, several other approaches have been tried and recent studies have shown that the selective serotonin reuptake inhibitors (SSRIs) can be useful in this context $[14,15]$.

The mechanism by which the SSRI's can reduce the incidence of hot flashes is not known, and so a clinical study was initiated to examine the hypothesis that the SSRI paroxetine might alter the metabolism of tamoxifen resulting in the production of less 4-
$\mathrm{OH}$-tam, and thereby reducing the incidence of hot flashes [4]. This hypothesis was based on the knowledge that paroxetine is metabolized by, and can inhibit the activity of CYP2D6, one of the enzymes believed to be responsible for the formation of 4-OHtam. No link was found between the incidence of hot flashes and the levels of any of the tamoxifen metabolites, but the study did show that the level of one chromatographic peak in the tamoxifen assays of patient plasma was highly sensitive to co-administration of paroxetine. This peak was determined to be a previously described minor tamoxifen metabolite: 4hydroxy $N$-desmethyl tamoxifen $[4,16,17]$. A small quantity of endoxifen was synthesized to verify the identity of the chromatographic peak and to evaluate the antiestrogenic activity of the metabolite. We further showed that endoxifen is almost exclusively generated by the action of CYP2D6 and that patients who carry a CYP2D6 genetic polymorphism that results in reduced enzyme activity, make very little endoxifen.

The antiestrogenic properties of endoxifen were not known, and so this study was initiated to characterize the ER binding properties and estrogen agonist/antagonist profile of the compound, as a first step toward determining the importance of this metabolite to the overall clinical response of patients undergoing tamoxifen therapy.

$N$-desmethyl tamoxifen has very similar properties to those of tamoxifen and the addition of the 4-hydroxyl group to tamoxifen dramatically increases the affinity of the compound for the ER. It is, therefore, not surprising that we find endoxifen to have essentially identical properties to 4-OH-tam with respect to receptor affinity and effects of cell proliferation and gene expression. The ER binding characteristics of endoxifen are indistinguishable from those of 4$\mathrm{OH}-\mathrm{tam}$ as are its estrogen antagonist properties as determined by the suppression of estrogen stimulated breast cancer cell proliferation. These findings are entirely consistent with what has recently been learned about the structural requirements for ER binding and antiestrogen properties based on the crystal structure of the ligand binding domain of the ER [18]. Although we have not conducted an exhaustive comparison of their actions in all organ systems where estrogen exerts some effect, based on these data is seems likely that 4-OH-tam and endoxifen are essentially functionally equivalent.

Data from our previous study [4], from earlier studies $[16,17]$, and from a study describing an as- 
say optimized for measuring endoxifen in patients samples [19], all show that endoxifen concentrations are notably higher than those of 4-OH-tam. More extensive ongoing studies have shown that plasma endoxifen concentrations are typically greater than those of 4-OH-tam when both drug and metabolite concentrations have reached steady state after 4 months of treatment with endoxifen concentrations being a mean of 6.8-fold greater (unpublished observations).

4-OH-tam has frequently been referred to in the literature as the active metabolite of tamoxifen, however, based on our present findings, if there is any validity to the concept of the predominance of a single active metabolite in response to tamoxifen therapy, then it is most likely endoxifen and not 4-OH-tam that is that metabolite. In reality it is more likely that it is the balance of the concentrations of all of the metabolites of tamoxifen along with the parent compound which mediate the clinical effects of the drug, but the fact remains that endoxifen is likely to play a significant role in this overall response. This realization is particularly important in light of our previous findings that show that plasma concentrations of endoxifen in patients are notably influenced by CYP2D6 genotype. There are more than 50 known genetic polymorphisms of this enzyme and roughly $7 \%$ of Caucasian patients do not express functional enzyme [20, 21]. Thus, it is possible that CYP2D6 genotype may have an important impact on patient response to tamoxifen since patients with defective CYP2D6 alleles have much lower plasma concentrations of endoxifen. We have recently described a facile method for genotyping archival paraffin imbedded tumor blocks for germline polymorphisms in several clinically important CYPs including CYP2D6 and we propose to attempt to address the question of the role of CYP2D6 genotype in tamoxifen response by interrogating historical clinical trials of tamoxifen efficacy [22]. Certain drugs that are known to inhibit the activity of CYP2D6 can mimic the effect of inactivating polymorphisms of the enzyme and reduce plasma concentrations of endoxifen [4]. This raises the possibility that co-administration of these agents might also alter patient response to tamoxifen therapy. Prospective clinical studies in which patients are genotyped for all of the relevant phases 1 and 2 metabolic enzymes and detailed records of concomitant medications are maintained will, however, be required in order to thoroughly evaluate the impact of tamoxifen metabolism on therapeutic outcome.

\section{Acknowledgements}

This study was supported in part by a Pharmacogenetics Research Network grant, U-01 GM61373 (D.F.). R.V.W. acknowledges the support of the American Cancer Society (IRG-58-006-41A) and the Purdue Cancer Center. We acknowledge the assistance of the Tissue Culture Shared Resource at the Lombardi Comprehensive Cancer Center which is supported in part by a Cancer Center Support Grant from the NCI (P30-CA51008).

\section{References}

1. American Cancer Society: Cancer Facts and Figures, 2002

2. Osborne CK: Tamoxifen in the treatment of breast cancer. $\mathrm{N}$ Engl J Med 339: 1609-1618, 1998

3. Furr BJ, Jordan VC: The pharmacology and clinical uses of tamoxifen. Pharmacol Ther 25: 127-205, 1984

4. Stearns V, Johnson MD, Rae JM et al.: Active tamoxifen metabolite plasma concentrations after coadministration of tamoxifen and the selective serotonin reuptake inhibitor paroxetine. J Natl Cancer Inst 95: 1758-1764, 2003

5. Jeppesen U, Gram LF, Vistisen K, Loft S, Poulsen HE, Brosen K: Dose-dependent inhibition of CYP1A2, CYP2C19 and CYP2D6 by citalopram, fluoxetine, fluvoxamine and paroxetine. Eur J Clin Pharmacol 51: 73-78, 1996

6. Leonessa F, Jacobson M, Boyle B, Lippman J, McGarvey M, Clarke R: Effect of tamoxifen on the multidrug-resistant phenotype in human breast cancer cells: isobologram, drug accumulation, and M(r) 170,000 glycoprotein (gp170) binding studies. Cancer Res 54: 441-447, 1994

7. Edwards DP, McGuire WL: 17 alpha-estradiol is a biologically active estrogen in human breast cancer cells in tissue culture. Endocrinology 107: 884-891, 1980

8. Bradford MM: A rapid and sensitive method for the quantitation of microgram quantities of protein utilizing the principle of protein-dye binding. Anal Biochem 72: 248-254, 1976

9. Johnson MD, Westley BR, May FE: Oestrogenic activity of tamoxifen and its metabolites on gene regulation and cell proliferation in MCF-7 breast cancer cells. Br J Cancer 59: 727-738, 1989

10. Jordan VC: Metabolites of tamoxifen in animals and man: identification, pharmacology, and significance. Breast Cancer Res Treat 2: 123-138, 1982

11. Patterson JS, Settatree RS, Adam AK, Kemp JV: Serum concentrations of tamoxifen and major metabolites during longterm Nolvadex therapy, correlated with clinical response. In: Mouridsen HT, Palshoff T (eds) Breast Cancer: Experimental and Clinical Aspects. Pergamon Press, Oxford, UK, 1980, p 89

12. Desta Z, Ward BA, Soukhova N, Flockhart DA: Comprehensive kinetic characterization of tamoxifen primary demethylation and hydroxylation by the human cytochrome P450s. Drug Metab Rev 25(Suppl. 2): 189 (abstract \#377), 2003

13. Fisher B, Costantino JP, Wickerham DL et al.: Tamoxifen for prevention of breast cancer: report of the National Surgical Adjuvant Breast and Bowel Project P-1 Study. J Natl Cancer Inst 90: 1371-1388, 1998 
14. Stearns V, Ullmer L, Lopez JF, Smith Y, Isaacs C, Hayes D: Hot flushes. Lancet 360: 1851-1861, 2002

15. Stearns V, Beebe KL, Iyengar M, Dube E: Paroxetine controlled release in the treatment of menopausal hot flashes: a randomized controlled trial. JAMA 289: 2827-2834, 2003

16. Lien EA, Solheim E, Kvinnsland S, Ueland PM: Identification of 4-hydroxy- $N$-desmethyltamoxifen as a metabolite of tamoxifen in human bile. Cancer Res 48: 2304-2308, 1988

17. Lien EA, Solheim E, Lea OA, Lundgren S, Kvinnsland S, Ueland PM: Distribution of 4-hydroxy- $N$-desmethyltamoxifen and other tamoxifen metabolites in human biological fluids during tamoxifen treatment. Cancer Res 49: 2175-2183, 1989

18. Shiau AK, Barstad D, Radek JT et al.: Structural characterization of a subtype-selective ligand reveals a novel mode of estrogen receptor antagonism. Nat Struct Biol 9: 359-364, 2002

19. Lee KH, Ward BA, Desta Z, Flockhart DA, Jones DR: Quantification of tamoxifen and three metabolites in plasma by high-performance liquid chromatography with fluorescence detection: application to a clinical trial. J Chromatogr B Analyt Technol Biomed Life Sci 791: 245-253, 2003

20. Marez D, Legrand M, Sabbagh $\mathrm{N}$ et al.: Polymorphism of the cytochrome P450 CYP2D6 gene in a European population: characterization of 48 mutations and 53 alleles, their frequencies and evolution. Pharmacogenetics 7: 193-202, 1997

21. Broly F, Gaedigk A, Heim M, Eichelbaum M, Morike K, Meyer UA: Debrisoquine/sparteine hydroxylation genotype and phenotype: analysis of common mutations and alleles of CYP2D6 in a European population. DNA Cell Biol 10: $545-558,1991$

22. Rae JM, Cordero KE, Scheys JO, Lippman ME, Flockhart DA, Johnson MD: Genotyping for polymorphic drug metabolizing enzymes from paraffin-embedded and immunohistochemically stained tumor samples. Pharmacogenetics 13: 501-507, 2003

Address for offprints and correspondence: Dr Michael D. Johnson, $\mathrm{PhD}$, Lombardi Comprehensive Cancer Center, Georgetown University Medical Center, Room W325, The Research Building, 3970 Reservoir Rd, NW, Washington, DC 20007, USA; Tel./Fax: +1-202-687-0217; E-mail: johnsom@georgetown.edu 